\title{
Design of low energy bunch compressors with space charge effects
}

\author{
A. He, F. Willeke, L. H. Yu, L. Yang, T. Shaftan, G. Wang, Y. Li, and Y. Hidaka \\ Photon Sciences Division, Brookhaven National Laboratory (BNL), Upton, New York 11973, USA \\ J. Qiang \\ Accelerator and Fusion Research Division, Lawrence Berkeley National Laboratory (LBNL), \\ Berkeley, California 94720, USA \\ (Received 15 August 2014; published 7 January 2015)
}

\begin{abstract}
In this paper, we explore a method to manipulate low energy electron bunches in a space charge dominated regime, and we use this method to design low energy linac bunch compressors to compress electron bunches in a space charge dominated regime. In the method, we use the space charge effects instead of avoiding them; i.e., we use the space charge forces to generate the required energy chirp instead of the ordinary method which uses the rf accelerating system to generate the chirp. We redefine the concepts of the dispersion function and beta functions in a space charge dominated regime to guide the optimization. Using this method, we study the low energy (5-22 MeV) linac bunch compressor design to produce short $(\sim 150 \mathrm{fs})$ and small size $(\sim 30 \mu \mathrm{m})$ bunches for the electron beam slicing project. The low energy linac bunch compressors work in a space charge dominated regime, and the bunches at the downstream of the gun have a negative energy chirp due to the space charge effects. To provide compression for the negative energy chirped bunch, we design a positive $R_{56}$ dispersive section using a four-dipole chicane with several quadrupole magnets. We have designed low energy linac bunch compressors with different photocathode rf guns. For example, one linac bunch compressor with the BNL photocathode electron rf gun has achieved a low energy bunch with the $166 \mathrm{fs}$ rms bunch length, 28 and $31 \mu \mathrm{m}$ rms beam size in the vertical and horizontal directions, respectively, at $5 \mathrm{MeV}$ with $50 \mathrm{pC}$ charge. Another example with LBNL's very-high frequency gun has achieved a low energy bunch with the $128 \mathrm{fs}$ rms bunch length, 42 and $25 \mu \mathrm{m}$ rms beam size in the vertical and horizontal directions, respectively, at $22 \mathrm{MeV}$ with $200 \mathrm{pC}$ charge.
\end{abstract}

DOI: 10.1103/PhysRevSTAB.18.014201

PACS numbers: 29.20.Ej

\section{INTRODUCTION}

Very short bunches of high-brightness electron beams with very high peak currents are required in many systems, e.g., free-electron laser (FEL) systems or collider systems. Usually, these bunches cannot be produced directly in guns, because space charge forces would destroy the brilliance within a short distance. Therefore, it is started with a low intensity bunch with a peak current of a few tens of amperes, accelerated to higher energy (i.e., $>100 \mathrm{MeV}$ ) where the space charge forces are weakened sufficiently by the $1 / \gamma^{2}$ scaling, and then compressing the bunch length to increase the peak current. Many magnetic bunch compressors have been designed to compress the accelerated high energy bunch to several hundred femtoseconds [1-5].

The electron beam slicing method [6] generates ultrashort $\mathrm{x}$-ray pulses using focused short low energy $(\sim 20 \mathrm{MeV})$ electron bunches to create short slices of

Published by the American Physical Society under the terms of the Creative Commons Attribution 3.0 License. Further distribution of this work must maintain attribution to the author(s) and the published article's title, journal citation, and DOI. electrons from the circulating electron bunches in a synchrotron radiation storage ring. When a low energy electron bunch crosses from the top of a high energy storage ring electron bunch, its Coulomb force will kick a short slice from the core of the storage ring electron bunch. The separated slices, when passing through an undulator, will radiate ultrashort $\mathrm{x}$-ray pulses at about $100 \mathrm{fs}$. In order to minimize the cost of the electron beam slicing system and to explore the lower limit of the compressor's bunch energy, we try to design a compressor without acceleration or with a short accelerating structure after the downstream of the rf gun to achieve the desired bunch compression and focusing. Therefore, we design the unconventional low energy (5-22 MeV) linac bunch compressors which work in the space charge effect dominated regime to obtain the required short $(\sim 150 \mathrm{fs})$, focused bunches $(\sim 30 \mu \mathrm{m})$ with 50-200 pC charge.

In the regime of strong space charge effects, two facts make the design of the low energy compressor difficult: (i) In the condition without space charge effects, the definition of parameters such as the longitudinal dispersion function, the transverse dispersion function, and the beta functions depends on the fact that the particles have constant energy. While in the regime with strong space 
charge effects, the particles' energy is no longer constant due to the space charge forces. Therefore, in the regime of strong space charge effects, these parameters are not well defined. (ii) The electron bunch blows up very quickly in a short propagation distance, e.g., in the dispersive section.

In this paper, we explore a method to systematically manipulate the low energy electron bunches in the space charge dominated regime, and we use this method to design low energy linac bunch compressors to compress the electron bunches in the space charge dominated regime.

The key ideas of this method to manipulate low energy electron bunches in the space charge dominated regime are as follows: We use the space charge effects instead of avoiding them to generate the energy chirp; we define a virtual reference particle whose six phase space coordinates are taken as the average values of all the bunch particles, and then with this definition we redefine the concepts of the dispersion function and beta functions in the space charge dominated regime to guide the optimization; we split the linac compressor into two sections, the gun section and the compressor section, and calculate the simulation of these two sections separately.

During the design, we follow the following procedures to optimize the low energy linac bunch compressor: (i) We first simulate the gun section with a desired space charge, and we save the bunch distribution at the downstream of the gun section in a file. (ii) We start the linear design of the compressor (the linear design means the design with space charge completely turned off). Here we use the conventional method and the basic parameters such as the energy chirp, the transverse dispersion, and the longitudinal dispersion, etc., to design the compressor section. (iii) We simulate the compressor section by reading the bunch distribution file generated in step (i) but deliberately set the space charge to zero. Then we gradually increase the space charge with small steps until it is increased to the desired space charge value. For each step, we tune the quadrupole magnets in the compressor to restore the redefined dispersion function and beta functions to be approximately the same as the case without space charge effects [i.e., the linear compressor obtained in step (ii)]. (iv) We combine together the gun section and the compressor section as one system. Then we carry out an optimization procedure using a genetic algorithm to optimize this system with space charge effects turned on. That is, during the optimization, in addition to the magnets in the compressor section, the parameters in the gun section (e.g., the gun phase, the solenoid strength, and the laser pulse length) are also varied.

In the following, we will use the method mentioned above to discuss in detail how we manipulate the space charge effects to compress and focus the bunch successfully in the space charge dominated regime. In Sec. II, we give a brief introduction about the linac bunch compressors we studied. In Sec. III, "the linear design stage," we discuss the requirements on the beta function, the dispersion function, and the linear longitudinal dispersion $R_{56}$ of the dispersive section and the requirement on the energy chirp of the bunch. We also discuss how to utilize the space charge effects to generate the desired negative energy chirp from the BNL photocathode electron rf gun [7], i.e., making the particles' energy at the head of the bunch higher than the tail. Then we use OPTIM [8] and ELEGANT [9] to perform the initial linear design of a magnetic chicane with positive linear longitudinal dispersion $R_{56}$ followed by a final focusing system, with space charge effects ignored. In Sec. IV, "the nonlinear design stage," we introduce a new concept of dispersion function and beta functions in the space charge dominated regime to guide the design and optimization. We apply PARMELA [10] to consider the space charge effects and carry out a multiobjective optimization procedure using the genetic algorithm to perform the global optimization [11]. To verify the simulation results, we benchmark the simulation results from the PARMELA with the results from the IMPACT-T [12] for our low energy linac bunch compressor. To study the coherent synchrotron radiation (CSR) effects, we compare the simulation results when CSR effects are turned on with those when CSR is turned off in the IMPACT-T in Sec. VA. In order to increase the repetition rate of the electron beam slicing system, in Sec. V B we change the linac compressor's rf gun from the BNL photocathode rf gun to LBNL's very-high frequency (VHF) gun [13] and redesign the linac electron bunch compressor by applying the IMPACT-T with both space charge effects and CSR effects considered.

\section{SCHEMATICS OF LINAC BUNCH COMPRESSORS}

To explore the method to compress a low energy electron bunch with high charge in a space charge effect dominated regime, we studied three linac electron bunch compressors at different energy with different guns by applying several particle simulation codes. The schematics of the linac electron bunch compressor discussed in the paper are shown in Fig. 1.

The first linac bunch compressor shown in Fig. 1(a) consists of the BNL photocathode electron rf gun [7] and a bunch compressor constructed by several magnets. This $6.75 \mathrm{~m}$ long linac bunch compressor is designed to compress an electron bunch with $50 \mathrm{pC}$ charge at $5 \mathrm{MeV}$. We will take the first linac bunch compressor as an example to discuss how to design a low energy bunch compressor with space charge effects in Secs. III and IV. Simulation codes OPTIM [8], ELEGANT [9], and PARMELA [10] are utilized to design the first linac bunch compressor.

In order to increase the kick angle for a better separation of the slice from the core [6], we need to increase the bunch charge and the bunch energy to keep the short longitudinal bunch length and small transverse beam size. Therefore, we add an accelerating section (i.e., a $0.95 \mathrm{~m}$ long SLAC-like cavity at $2.856 \mathrm{GHz}$ ) between the BNL photocathode 
(a)

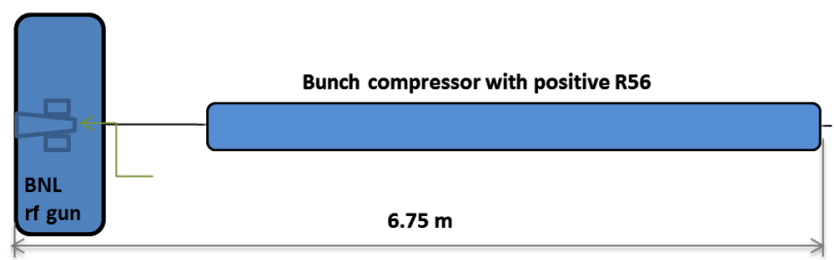

(b)

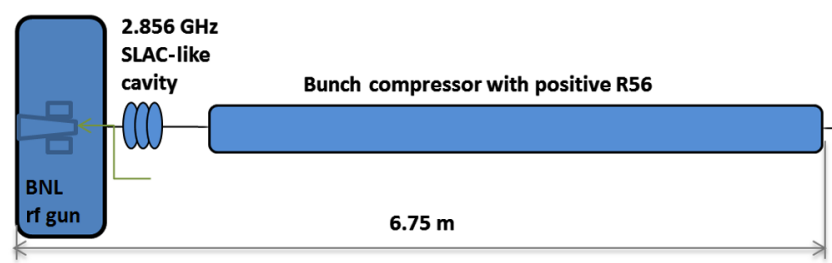

(c)

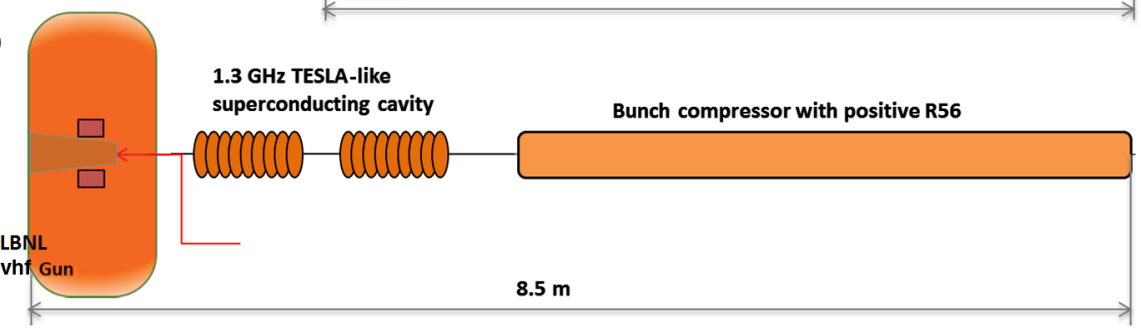

FIG. 1. Schematics of linac bunch compressors. (a) consists of the BNL photocathode rf gun and a bunch compressor constructed by several magnets. (b) consists of the BNL photocathode rf gun, a SLAC-like cavity at $2.856 \mathrm{GHz}$, and a bunch compressor. (c) consists of LBNL's VHF gun, two TESLA-like superconducting cavities at $1.3 \mathrm{GHz}$, and a bunch compressor.

electron rf gun and the bunch compressor as shown in Fig. 1(b). The second linac bunch compressor works to compress a bunch with charge from 100 to $150 \mathrm{pC}$ at $12 \mathrm{MeV}$. We apply tracking code PARMELA to design the second linac bunch compressor. Then we apply tracking code IMPACT-T [12] to benchmark the simulation results obtained by the code PARMELA and to consider the effects of CSR.

In order to increase the repetition rate of the electron beam slicing system to increase the photon flux [6], we designed the third linac bunch compressor as shown in Fig. 1(c), which consists of LBNL's VHF gun [13] (it operates at $186 \mathrm{MHz}$ with a repetition rate of $1 \mathrm{MHz}$ ), two $1.32 \mathrm{~m}$ long $1.3 \mathrm{GHz}$ TESLA-like superconducting cavities [14], and a bunch compressor. The third linac bunch compressor works to compress a bunch with charge $200 \mathrm{pC}$ at $22 \mathrm{MeV}$. Simulation code IMPACT-T is utilized to design this linac bunch compressor.

\section{LINEAR DESIGN OF THE COMPRESSOR WITH POSITIVE $\boldsymbol{R}_{56}$ CHICANE}

In the space charge dominated regime, due to the strong space charge forces, the particles' energy changes as it passes through the compressor. Therefore, in the space charge dominated regime, there is no particle suitable to be used as a reference particle, whose energy is required to be constant. In this paper, we define a virtual reference particle whose six phase space coordinates are taken as the average values of all the particles. With this definition, the six phase space coordinates of the bunch particles are referred to as the virtual reference particle's. For example, the particle's arrival time $t$ is the relative time of flight with respect to the virtual reference particle's arrival time for an observation point, and the head particles of the electron bunch correspond to $t<0$. The bunch's energy chirp $h$ is defined by $h=d \delta /\left.c d t\right|_{t=0}$, where the bunch's energy spread $\delta$ is a function of bunch particle position $t$. Therefore, a negative energy chirped bunch with $h<0$ means the energy of the head particles with $t<0$ is larger than the tail particles'. The linear longitudinal dispersion $R_{56}$ of a dispersive section is defined by $R_{56}=d l / d \delta$, where $l$ is the path length difference for particles with a relative energy spread $\delta$.

With the above definitions, we will discuss the linear design of the compressor in this section. In Sec. III A, we give the relation of the bunch's energy chirp $h$ and the linear longitudinal dispersion $R_{56}$ of a dispersive section to obtain the maximum compression. In Sec. III B, we analyze the energy chirp of the bunch at the downstream of the BNL photocathode $\mathrm{rf}$ gun and find a large positive $R_{56}$ (i.e., $170 \mathrm{~mm}$ ) is required for the dispersive section. In Sec. III C, we design an unconventional four-dipole chicane with positive $R_{56}$ by adding several quadrupole magnets in the dipole magnets to compress the negative energy chirped bunch. In Sec. IIID, with the final compressed bunch length of $100 \mathrm{fs}$ as a target, we estimate the limitations on the beta function, the dispersion function, and $R_{56}$ of the compressor. The analysis in Sec. III D shows that we need to control $R_{56}$ of the dispersive dipoles less than $84 \mathrm{~mm}$ and increase the bunch's energy chirp larger than $1 \% / \mathrm{ps}$. In Sec. III E, we use the space charge effects to increase the negative energy chirp and then to reduce the required $R_{56}$ 
of the dispersive section. The linear simulation results are shown in Sec. III F.

During the linear compressor design with space charge effects ignored, we iterate over the process of the simulation and estimates as described by Secs. III B and III E until the result is convergent. The estimated limitations on the maximum value of the beta function and dispersion function are used as a guideline during the design and are confirmed later by simulation. To make the analysis of the estimates more explicit, the parameters used in these estimated examples come from the result of the convergent linear simulation.

\section{A. Relation of the bunch's energy chirp $\boldsymbol{h}$ and $\boldsymbol{R}_{\mathbf{5 6}}$}

To compress an energy chirped bunch longitudinally, depending on whether the energy chirp of the bunch is positive or negative, the flight time through some dispersive section must be shorter or longer for the tail of the bunch than it is for the head. In linear approximation, the compressed rms bunch length $\sigma_{1}$ is simply scaled from its original rms bunch length $\sigma_{0}$ as [15]

$$
\sigma_{1} \approx \sqrt{\left(1+h R_{56}\right)^{2} \sigma_{0}^{2}+R_{56}^{2} \sigma_{\delta}^{2}}
$$

where $h$ is the energy chirp, $\sigma_{\delta}$ is the rms relative intrinsic energy spread, and $R_{56}$ is the linear longitudinal dispersion of the dispersive section. This equation shows that, to maximize compression, the $R_{56}$ should match with the bunch's energy chirp $h$ by

$$
R_{56}=-1 / h \text {. }
$$

\section{B. Energy chirp downstream of the BNL rf gun}

Usually, the chirp is generated by introducing a correlation between the longitudinal position of the particles in the bunch and their energy by using a rf accelerating system. After the rf accelerating system, these bunches' energy chirp is usually positive $(h>0)$ that means the head particle's energy is lower than the tail particle's.

In our case, there is no accelerating system to generate the positive energy chirp. By simulation, we confirm that, no matter how we adjust the phase of the rf gun, the energy chirp from the BNL photocathode electron rf gun is always small and negative $(h<0)$ due to the space charge effects, i.e., the head particle with higher energy. For example, in the bottom-left plot of Fig. 2, the $50 \mathrm{pC}$ charged bunch is chirped by the space charge forces to be a small negative energy chirp $-0.25 \% / \mathrm{ps}$, i.e., $1 / h=-120 \mathrm{~mm}$, downstream of the rf gun. To compress such a negative chirped bunch, the dispersive section must have positive $R_{56}=$ $120 \mathrm{~mm}$ such that the higher energy particles go through a longer path length than the low energy particles do. The bunch is compressed after passing through the dispersive section. For our $5 \mathrm{MeV}$ low energy linac compressor with a
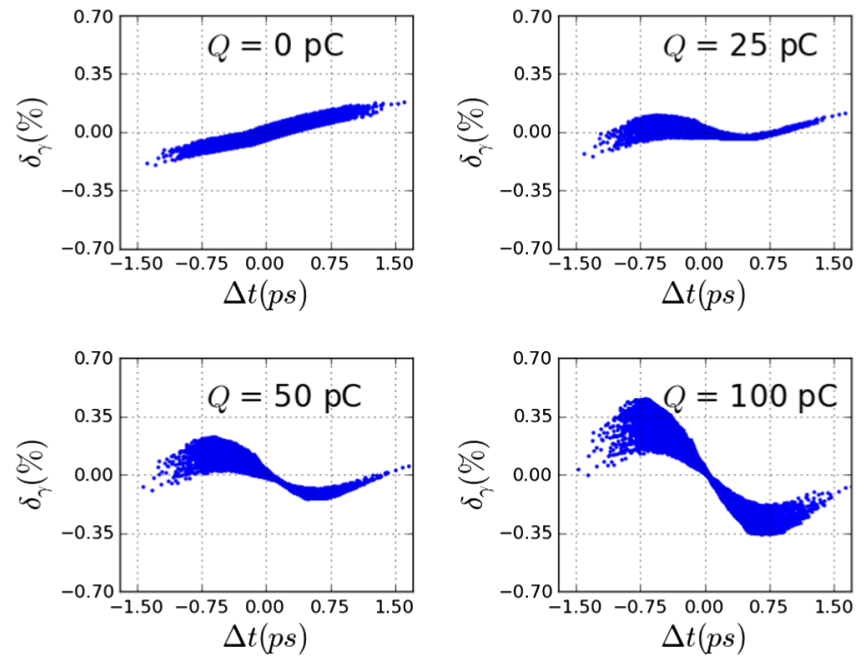

FIG. 2. Negative energy chirp downstream of the BNL photocathode rf gun increases with the increase of the bunch charge due to the increase of space charge effects. Simulation results come from PARMELA. In these plots, particles located at $\Delta t>0$ correspond to the tail particles of the bunch.

$5 \mathrm{~m}$ long chicane, the $R_{56}=-s / \gamma^{2}=-50 \mathrm{~mm}$ induced by the drift space must be included. Therefore, to maximize compression, the $R_{56}$ contributed from the dispersive elements, i.e., dipoles in the dispersive section, is $170 \mathrm{~mm}$.

\section{Positive $\boldsymbol{R}_{56}$ chicane}

The simplest and most common magnetic compressor is the four-dipole magnetic chicane as shown in the top plot of Fig. 3. This ordinary chicane with negative linear longitudinal dispersion $\left(R_{56}<0\right)$ is designed to compress a bunch with positive energy chirp $(h>0)$, i.e., head particles with lower energy and tail particles with higher energy.
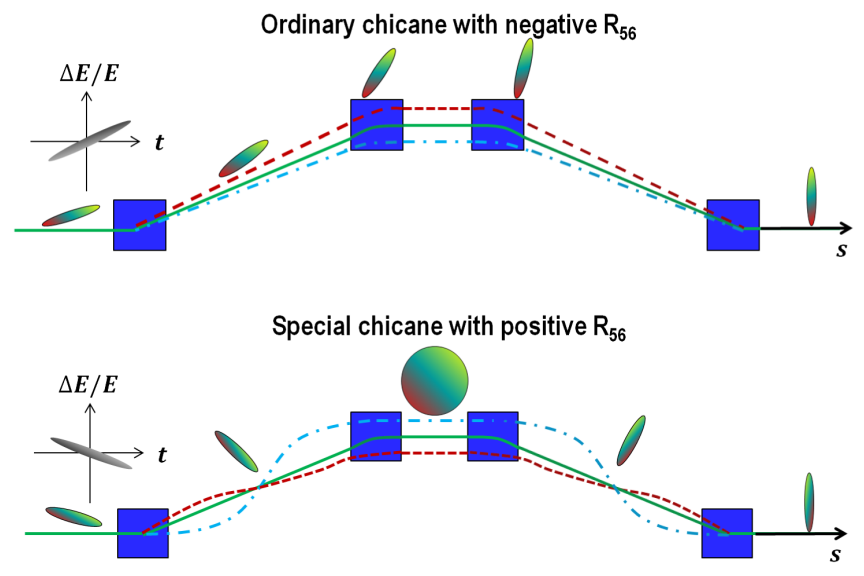

FIG. 3. Schematic layout of the ordinary negative $R_{56}$ chicane (top plot) and the special positive $R_{56}$ chicane (bottom plot). The blue, green, and red curves represent the trajectories of high energy particle, center energy particle, and low energy particle, respectively. 


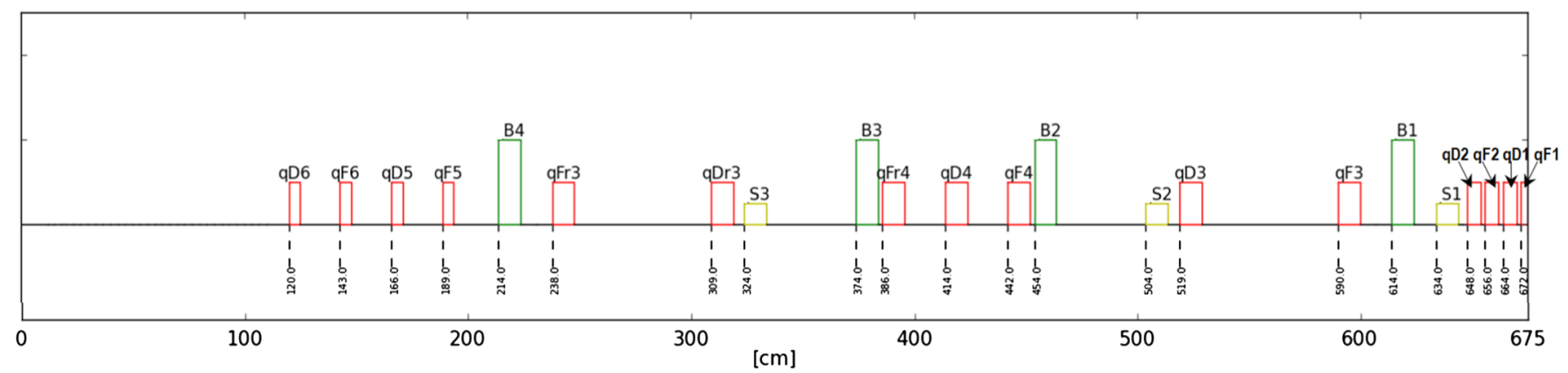

FIG. 4. The lattice configuration of the positive $R_{56}$ compressor. The green columns indicate the four dipole magnets, the red columns indicate the quadrupole magnets, and the yellow ones indicate the sextupole magnets. The 0 point corresponds to the starting point of the photocathode rf gun.

Quadrupole magnets and sextupole magnets are commonly introduced between the dipoles of the four-dipole chicane to achieve specific functions $[16,17]$. In our case, simulation analysis in Sec. III B shows that the bunch in our case with negative energy chirp $(h<0)$ cannot be compressed by the ordinary negative $R_{56}$ chicane. We design a special chicane whose linear longitudinal dispersion is positive $R_{56}>0$ to compress the negative energy chirped bunch.

The schematic layout of our positive $R_{56}$ chicane is shown in the bottom plot of Fig. 3. It is constructed by four rectangular dipole magnets each with length $L_{\text {dipole }}=$ $0.1 \mathrm{~m}$ and bending angle $\theta=20^{\circ}$. Between the dipoles, we set several quadrupole magnets to flip the particles' trajectories so that the higher energy particles' path length (the blue dashed curve in the bottom plot of Fig. 3) is longer than the lower's (the red dashed curve in the bottom plot of Fig. 3). During the chicane, it is possible for the tail lower energy particle to catch up with the head higher energy particle. Then the negative energy chirped bunch can be compressed to a short bunch length.

The lattice configuration of the compressor is illustrated in Fig. 4. The green columns indicate the four bending dipole magnets, the red columns indicate the quadrupole magnets, and the yellow ones indicate the sextupole magnets. The four matching quadrupole magnets before dipole magnet $B 4$ are used to match the Twiss parameters of the beam downstream of the rf gun with the Twiss parameters of the beam upstream of the chicane. The quadrupole magnets between the dipole magnets are set to flip the particles' trajectories. The other four focusing quadrupole magnets after dipole magnet $B 1$ are used to focus the beam to very small size. In the electron beam slicing method [6], quadrupole magnet $q F 1$ in Fig. 4 will be located in the tunnel of the storage ring, and the end point of $q F 1$ is the interaction point of the storage ring bunch and the linac bunch.

The $R_{56}$ of the chicane mainly comes from the dipoles' dispersion by $R_{56}=\int \frac{\eta}{\rho} d s \sim \eta \theta_{i}$. This equation shows that, for a fixed $\theta_{i}$, the estimated value of the linear longitudinal dispersion $R_{56}$ is determined by $\eta$ at the edges of the dipole magnets. To obtain a positive $R_{56}$, we use several quadrupole magnets to flip the particles' trajectories to change the sign of $\eta$ at the edges of the dipole magnets. For an example as shown in Fig. 5, $\eta$ is changed from $\eta<0$ downstream of $B 4$ (located at $224 \mathrm{~cm}$ in Fig. 4) to $\eta>0$ upstream of $B 3$ (located at $374 \mathrm{~cm}$ in Fig. 4) by using the quadrupole magnets $q F r 3$ and $q D r 3$.

To obtain a large positive $R_{56}$ with fixed $\theta_{i}$, we need to increase the dispersion $\eta$ at the edges of dipole magnets $B 2$ and $B 3$. According to the analysis of Sec. III D, to compress the bunch length lower than $100 \mathrm{fs}$, we need to control the dispersion. At the same time, we know the maximum of
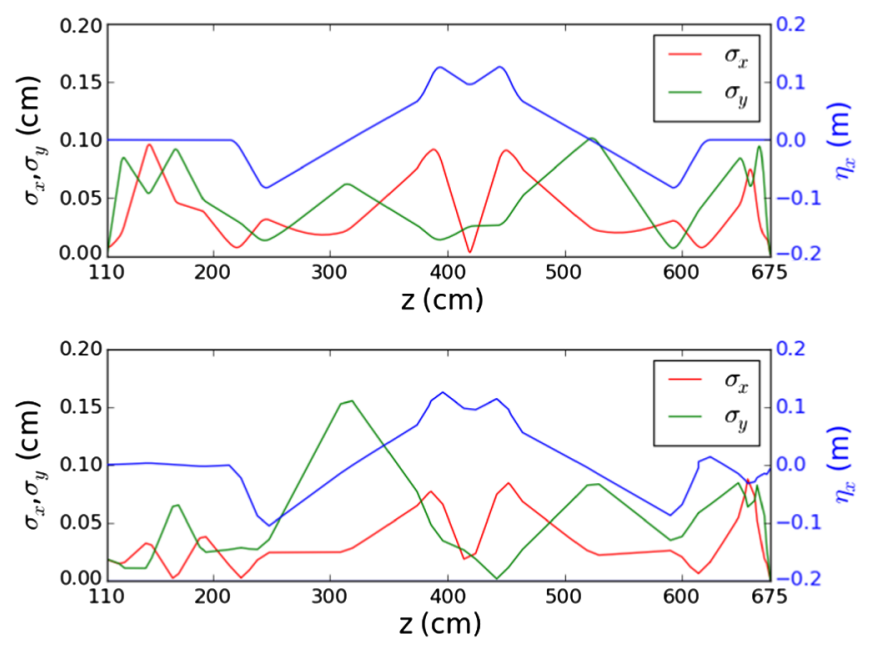

FIG. 5. The rms beam size and dispersion function from the first matching quadrupole magnet to the last focusing quadrupole magnet without space charge effects from OpTIM (top plot) and with space charge effects from PARMELA (bottom plot). To calculate the redefined dispersion (the detailed discussion about the redefined dispersion can be found in Sec. IV A) in the bottom plot, we select those particles whose energy deviation upstream of the first matching quadrupole magnet (i.e., qD6) at $110 \mathrm{~cm}$ is in the range $0.7 \%-0.8 \%$. To calculate the redefined beta functions (actually, it is the beam size in the plot) in the bottom plot, we select those particles whose emittance upstream of the first matching quadrupole magnet (i.e., qD6) at $110 \mathrm{~cm}$ is smaller than $0.1 \mathrm{~mm}$ mrad. 
dispersion $\eta$ is located at quadrupole magnets, i.e., $q F r 4$ and $q F 4$. Therefore, to increase the $R_{56}$ contributed from $B 2$ and $B 3$ without increasing the dispersion between the two middle dipole magnets, we move quadrupole magnets very close to the dipole magnets shown in Fig. 4 (i.e., the quadrupole magnet $q F r 4$ is very close to the second dipole magnet $B 3$, and the quadrupole magnet $q F 4$ is very close to the third dipole $B 2$ ). With such a setting, $\eta$ does not continue to increase once the beam passes through the dipole magnet $B 3$; i.e., $\eta$ almost reaches its maximum downstream of the dipole magnet $B 3$.

\section{Requirements of beta function, dispersion function, and $\boldsymbol{R}_{\mathbf{5 6}}$}

Both the betatron oscillation and the dispersion cause path length difference and lead to bunch length increase. In this subsection, through some linear estimates based on our linear simulation, we discuss the influences of betatron oscillation and the dispersion on the bunch length. With the final bunch length of $100 \mathrm{fs}$ as a target, we calculate the requirements of the beta function $\beta$, the dispersion function $\eta$, and the linear longitudinal dispersion $R_{56}$ of the dispersive elements for the linac bunch compressor.

\section{Requirement of beta function}

We assume there are two particles traveling through a lattice section with a longitudinal distance of $l$. If one particle travels on the axis, and if the other one's transverse location varies from 0 to $\sigma$ after the longitudinal traveling of the distance $l$, then, as an estimate, the path length difference between the two particles is approximately expressed as

$$
\Delta l=\sqrt{l^{2}+\sigma^{2}}-l \approx \frac{\sigma^{2}}{2 l}=\frac{\beta \varepsilon}{2 l},
$$

where $\beta$ is the beta function and $\varepsilon$ is the emittance. This equation shows that the large betatron oscillation causes a long path length and hence a longer bunch length. For our compressor, during the final focusing section, the beam size experiences a large change. We assume for the final focusing section $l=0.5 \mathrm{~m}, \varepsilon=1 \mu \mathrm{m}$, and $\beta=10 \mathrm{~m}$, and then the path length difference is $\Delta l=10 \mu \mathrm{m}$. With $10 \mu \mathrm{m}$, i.e., $30 \mathrm{fs}$ path length difference, the bunch length of a $100 \mathrm{fs}$ bunch increases to around $\sqrt{(100 \mathrm{fs})^{2}+(30 \mathrm{fs})^{2}}=105 \mathrm{fs}$. Based on the estimation, for the linear simulation with the space charge effects ignored, in order to be sure the path length increase due to betatron oscillation is negligible, we need to control the beta function within $10 \mathrm{~m}$.

\section{Requirement of dispersion function}

The path length of an electron particle in the FrenetSerret coordinate system with the vertical contribution $z^{\prime}$ ignored is [18]

$$
\begin{aligned}
l & =\int_{0}^{L} \sqrt{\left(1+\frac{x}{\rho}\right)^{2}+x^{\prime 2}} d s \\
& \approx l_{0}+\int_{0}^{L}\left[\frac{x}{\rho}+\frac{1}{2}\left(\frac{x}{\rho}\right)^{2}+\frac{1}{2}\left(x^{\prime}\right)^{2}\right] d s,
\end{aligned}
$$

where $\rho$ is the bending radius of a dipole and $l_{0}$ is the orbit length of the unperturbed orbit. By using the dispersion function $\eta$ and its derivative $\eta^{\prime}$, the particle's horizontal position $x$ and its derivative $x^{\prime}$ are expressed as $x=\eta \delta$ and $x^{\prime}=\eta^{\prime} \delta$, respectively, where $\delta$ is the energy spread. Then the path length difference of the electron particles induced by the dispersion is written as

$$
\begin{aligned}
\Delta l & \approx \int_{0}^{L}\left[\frac{\eta}{\rho} \delta+\frac{1}{2}\left(\frac{\eta}{\rho} \delta\right)^{2}+\frac{1}{2}\left(\eta^{\prime} \delta\right)^{2}\right] d s \\
& =R_{56} \delta+\int_{0}^{L} \frac{1}{2}\left(\frac{\eta}{\rho} \delta\right)^{2} d s+\int_{0}^{L} \frac{1}{2}\left(\eta^{\prime} \delta\right)^{2} d s,
\end{aligned}
$$

where $L$ is the length of the dispersive section. The coefficient $R_{56}=\int_{0}^{L} \frac{\eta}{\rho} d s$ is the linear longitudinal dispersion and is usually the leading term for the bunch compression.

The first term on the right side of Eq. (4), i.e., $R_{56} \delta$, is designed to make the linear longitudinal dispersion $R_{56}$ match with the bunch's energy chirp $h$ upstream of the dispersive section through Eq. (2). In the following, under the condition of a small relative energy spread $(\delta<2 \%)$, we estimate the contributions of the second term and the third term on the right side of Eq. (4) on the path length difference. The estimates based on our linear simulation show that, to control the bunch length smaller than $100 \mathrm{fs}$, the dispersion function is required to be less than $12 \mathrm{~cm}$.

To estimate the bunch length increase induced by the second term on the right side of Eq. (4), i.e., $\int_{0}^{L} \frac{1}{2}\left(\frac{\eta}{\rho} \delta\right)^{2} d l$, we assume the dispersion and the energy spread during dipole are $\eta=0.07 \mathrm{~m}$ and $\delta=1 \%$, respectively. According to the linear lattice design in Sec. III C, the bending radius of the dipole is $\rho=0.286 \mathrm{~m}$ with $L_{\text {dipole }}=$ $0.1 \mathrm{~m}$ and $\theta=20^{\circ}$. From our simulation, we find the contribution of the dispersion mainly comes from the second and the third dipoles of the chicane, i.e., $B 2$ and $B 3$ in Fig. 4. For the estimation, we ignore the bunch length increase due to the nonlinear dispersion influences of the first and fourth dipoles, i.e., $B 1$ and $B 4$ in Fig. 4. Therefore, the bunch length increase induced by the dispersion $\eta$ is

$$
\int_{0}^{L} \frac{1}{2}\left(\frac{\eta}{\rho} \delta\right)^{2} d l \approx \frac{1}{2}\left(\frac{\eta}{\rho} \delta\right)^{2} \times 2 L_{\text {dipole }} \approx 0.6 \mu \mathrm{m} .
$$

For our case, the bunch length increase due to the dispersion is about $2 \mathrm{fs}$. It is very small and can be ignored.

To estimate the effects of the derivative of the dispersion $\eta^{\prime}$ on the bunch length increase, according to our simulation, we assume the dispersion varies from the minimum 
$\eta_{\min }=-12 \mathrm{~cm}$ to the maximum $\eta_{\max }=12 \mathrm{~cm}$ during a dispersion section of $2 \mathrm{~m}$. Then the derivative of the dispersion is $\eta^{\prime}=0.12$. Without the consideration of space charge effects, the four-dipole chicane is symmetric. Therefore, we assume the total length of the dispersion section is $L=4 \mathrm{~m}$. The bunch length increase induced by the derivative of the dispersion $\eta^{\prime}$ is estimated as

$$
\int_{0}^{L} \frac{1}{2}\left(\eta^{\prime} \delta\right)^{2} d l \approx \frac{1}{2}\left(\eta^{\prime} \delta\right)^{2} \times L \approx 3 \mu \mathrm{m}
$$

This corresponds to a bunch length increase of $10 \mathrm{fs}$. The previous estimate indicates that the bunch length increase induced by dispersion mainly comes from the derivative of the dispersion $\eta^{\prime}$. Therefore, to control the bunch length smaller than $100 \mathrm{fs}$, we require the dispersion function to be less than $12 \mathrm{~cm}$.

About the influence of $\delta^{2}$ on the bunch length increase, we point out that, when the relative energy spread $\delta$ becomes very large, a second-degree parabolic half-moon-shaped particle distribution appears in the longitudinal phase space of the bunch as shown in Fig. 11(b). When $\delta^{2}$ dominates the contribution on the bunch length increase, to reduce the bunch length, we need to decrease the relative energy spread of the bunch before the chicane as discussed in Sec. V B.

\section{Requirement of linear longitudinal dispersion $\boldsymbol{R}_{\mathbf{5 6}}$}

The linear longitudinal dispersion of a four-dipole chicane except the drift space is approximately expressed as $R_{56}=\int \frac{\eta}{\rho} d s \approx 2 \eta \theta$, where we ignore the contribution of the first and the second dipole due to the small dispersion function. In our design, to control the maximum of dispersion during the chicane, we move the quadrupoles between the two middle dipoles very close to the second and third dipoles, respectively, as discussed in Sec. III C. For such a chicane with $\eta_{\max }=12 \mathrm{~cm}$, the estimated linear longitudinal dispersion from the dispersive dipoles is $R_{56} \approx 2 \eta_{\max } \theta=2 \times 12[\mathrm{~cm}] \times \frac{\pi}{9}=84 \mathrm{~mm}$. When the contribution from the drift space $R_{56}=-50 \mathrm{~mm}$ is included (see the explanation in Sec. III B), the total $R_{56}$ for the dispersive section is $34 \mathrm{~mm}$. Therefore, according to Eq. (2), a dispersive section with $R_{56}=34 \mathrm{~mm}$ corresponds to compressing a bunch with energy chirp about $1 / h=-34 \mathrm{~mm}$; i.e., $h$ is $-1 \% / \mathrm{ps}$. Based on this, we conclude that, in order to control $R_{56}$ of the dispersive dipoles less than $84 \mathrm{~mm}$, we need the absolute value of the energy chirp near or larger than $1 \% / \mathrm{ps}$.

\section{E. Use space charge effects to introduce the required negative energy chirp}

Based on the discussion in Sec. III B, to compress the small negative energy chirped bunch $(h=-0.25 \% / \mathrm{ps})$ downstream of the BNL photocathode electron rf gun, we need a dispersive section with a large $R_{56}=170 \mathrm{~mm}$ coming from the dispersive dipole magnets, while in Sec. III D, we know the large $R_{56}$ requirement leads to a large dispersion function, large beta functions, and a large beam size which will induce a large betatron oscillation. And then the large betatron oscillation causes a longer path length and hence a longer bunch length. Therefore, with the space charge effects ignored, to control the compressed bunch length to be less than $100 \mathrm{fs}$, the $R_{56}$ of the dispersive dipole magnets need to be controlled to be less than $84 \mathrm{~mm}$ which corresponds to compressing a bunch with energy chirp $1 / h=-34 \mathrm{~mm}$, i.e., $-1 \% / \mathrm{ps}$. Analysis shows that, to compress the negative energy chirped bunch from the BNL photocathode electron rf gun to $100 \mathrm{fs}$ bunch length, we need to increase the bunch's energy chirp from $-0.25 \% / \mathrm{ps}$ to $-1 \% / \mathrm{ps}$.

From our simulation, we find that the negative energy chirp of the bunch increases with the increase of the bunch charge due to the space charge forces increasing with the increase of bunch charge. Figure 2 shows the influence of space charge forces on the bunch's energy chirp downstream of the BNL photocathode rf gun.

To reduce the $R_{56}$ of the dispersive section, instead of avoiding space charge forces, we focus the electron bunch to strengthen the space charge forces by increasing the strength of the focusing magnets as shown in Fig. 4. Because of the space charge forces, the head particles' energy are increased and the tail particles' energy are decreased. Then the bunch's energy chirp is increased from $-0.25 \% / \mathrm{ps}$ downstream of the rf gun as shown in the left of Fig. 6 to $-1.5 \% /$ ps upstream of the first matching quadrupole magnet (i.e., $q D 6$ ) as shown in the right of Fig. 6. That means $1 / h$ is reduced from $-120 \mathrm{~mm}$ downstream of the $\mathrm{rf}$ gun to $-20 \mathrm{~mm}$ upstream of the chicane. Thus, according to Eq. (2) and with the consideration of $R_{56}=-50 \mathrm{~mm}$ due
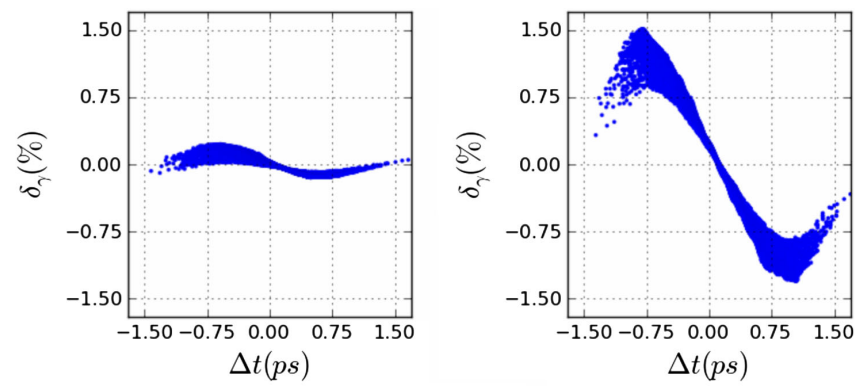

FIG. 6. Longitudinal phase spaces at 12 (left) and $110 \mathrm{~cm}$ (right) of the linac compressor in Fig. 4. The left phase space corresponding to energy chirp $1 / h=-120 \mathrm{~mm}$ is recorded downstream of the photocathode $\mathrm{rf}$ gun, and the right phase space corresponding to energy chirp $1 / h=-20 \mathrm{~mm}$ is recorded upstream of the first matching quadrupole magnet (i.e., qD6). Bunch charge in the simulation is $50 \mathrm{pC}$. We focus the bunch to strengthen the space charge forces and then to increase the energy chirp from $-0.25 \% / \mathrm{ps} \quad(1 / h=-120 \mathrm{~mm})$ to $-1.5 \% / \mathrm{ps}$ $(1 / h=-20 \mathrm{~mm})$. In these plots, particles located at $\Delta t>0$ correspond to the tail particles of the bunch. 
to drift space, the required $R_{56}$ of the dispersive dipoles is greatly reduced to 70 from $170 \mathrm{~mm}$.

\section{F. Simulation result of linear design}

The simulation codes used in the linear design include OptiM [8], ElEGANT [9], and PARMELA [10]. Because the $R_{56}=-s / \gamma^{2}$ induced by drift space is not included in ELEGANT, we use OptiM for the linear design of the compressor section. Because we know the design goal at the final point of the compressor (i.e., the final beam size and bunch length), we start the design in the reverse direction, i.e., from the final focusing point (located at $675 \mathrm{~cm}$ in Fig. 4) to upstream of the chicane (located at $214 \mathrm{~cm}$ in Fig. 4). We apply PARMELA to obtain the Twiss parameters of the beam downstream of the rf gun with space charge effects turned on. We apply ELEGANT to match the Twiss parameters of the beam downstream of the rf gun (obtained by simulation code PARMELA) with the Twiss parameters of the beam upstream of the chicane (obtained by simulation code ОРтіM).

During the linear compressor design, we iterate over the process of simulation and estimates as described by Secs. III B-III E until the result is convergent. We obtain the linear lattice bunch length of $100 \mathrm{fs}$ and the transverse beam size of $30 \mu \mathrm{m}$ at the final point of the compressor with space charge effects neglected. The beam size and the dispersion function of our linear compressor without space charge effects are well controlled within the limit we specified, as shown in Fig. 5 (top plot).

\section{NONLINEAR OPTIMIZATION WITH SPACE CHARGE EFFECTS}

\section{A. Dispersion function and beta functions in space charge regime}

The space charge effects simulation study of the compressor system consisting of only a BNL photocathode rf gun and a compressor chicane with a matching section is carried out by the code PARMELA [10]. PARMELA is a scalar code developed in Los Alamos. This simulation code can track relativistic particles taking into account space charge effects in the 6D space. In the simulation, we assume the field gradient is $100 \mathrm{MV} / \mathrm{m}$ at the cathode and fix the laser spot size at a radius of $2 \mathrm{~mm}$.

When the space charge effects are turned on, two facts make the nonlinear optimization difficult: (i) Because of the strong space charge forces, the particle energy is no longer constant, and then the dispersion function and beta functions both lose their original meaning. (ii) The beam blows up very quickly with the increase of the bunch charge.

To provide a guidance for the optimization, we define the virtual reference particle as explained in Sec. III, and then we redefine the equivalent dispersion by averaging the particles' trajectory in selected initial energy ranges and the equivalent beta functions using rms beam size in the selected initial emittance range. An example is shown in the bottom plot of Fig. 5. To calculate the equivalent dispersion (the blue curve in the bottom plot of Fig. 5), we select those particles whose energy deviation upstream of the first matching quadrupole magnet (i.e., $q D 6$ ) at $110 \mathrm{~cm}$ is in the range $0.7 \%-0.8 \%$, and then we average these selected particles' trajectory along the lattice. The averaged trajectory of these selected particles is the equivalent dispersion along the lattice. To calculate the equivalent beta functions (the red and the green curves in the bottom plot of Fig. 5; actually, it is beam size in the plot), we select those particles whose emittance upstream of the first matching quadrupole magnet (i.e., $q D 6$ ) at $110 \mathrm{~cm}$ is smaller than $0.1 \mathrm{~mm} \mathrm{mrad}$, and then we calculate these selected particles' rms beam size as the equivalent beta functions.

To avoid the beam blowing up, we split the linac compressor into two sections, the gun section and the compressor section, and calculate the simulation of these two sections separately. We start the simulation of the compressor section by reading the bunch distribution file downstream of the gun section but deliberately setting the space charge to zero. Then we gradually increase the space charge with small steps until it is increased to the desired space charge value. For each step, with the guidance of the redefined functions we manually adjust those quadrupole magnets in the compressor to restore the redefined dispersion function and beta functions to be approximately the same as the case without space charge effects, i.e., the linear compressor obtained in Sec. III. This trial and error procedure allows us to gradually increase charge from 0 to $30 \mathrm{pC}$ without blowing up the beam, without losing particles.

Figure 5 shows the linear lattice functions along the compressor without space charge effects from the code OptiM (top) and the nonlinear redefined functions with space charge effects from code PARMELA (bottom). It is seen that the effective dispersion function and beam size are restored to the similar forms as the linear functions. It shows the chicane still has the symmetrical behavior in the space charge force dominated regime approximately. Even though the bunch length is still very large (about $700 \mathrm{fs}$ ), this provision makes it possible to carry out optimization by a genetic algorithm without losing particles during the tracking.

\section{B. Global optimized results}

We carry out a multiobjective optimization procedure using a genetic algorithm [11]. In the optimization we set more than 20 variables which include the laser pulse length, laser phase relative to rf gun phase, solenoid strength, field strengths of quadrupole magnets and dipole magnets in the chicane, field strengths of matching quadrupole magnets, and field strengths of final focusing quadrupole magnets. The optimized objects are the bunch length and the sum of transverse rms beam sizes. When we use the genetic 
algorithm, we iterate by alternating optimization with adjustment of the variable scanning limits. This procedure reduced the variable scanning range and, hence, is crucially important for the control of the phase space size to be studied for each stage of the optimization step. We also gradually increase charge from 30 to $50 \mathrm{pC}$ by the genetic algorithm.

At $5 \mathrm{MeV}$ with $50 \mathrm{pC}$ charge, the optimization leads to $166 \mathrm{fs}$ rms bunch length with 28 and $31 \mu \mathrm{m}$ for horizontal and vertical rms beam size, respectively, at the focal point. The optimized results are given in Table I. The rms bunch length is calculated for $90 \%$ of particles, with $10 \%$ longitudinal tail cutoff. The particles which are cut off are those particles which have the largest longitudinal position deviation from the average longitudinal position of the bunch at the final point of the linac compressor. When the number of simulation particles is increased to 10000 , the result is convergent.

In the electron beam slicing method [6], we need to further reduce the slice pulse length and to increase the kick angle for a better separation of the slice from the core, so we need to increase the bunch charge and the bunch energy to keep the short longitudinal bunch length and small transverse beam size. We utilize the simulation result for a $5 \mathrm{MeV}$ linac compressor [i.e., the first linac compressor shown in Fig. 1(a)] as a basis to study $12 \mathrm{MeV}$ cases by adding an accelerating section as shown in Fig. 1(b) and gradually increasing the rf amplitude during the global optimization. The increase of beam energy allows the increase of charge while maintaining the required small beam size and bunch length at the point of the linac bunch compressor. The optimized results are given in Table II.

\section{Analysis of one optimized example}

Figure 7 shows the evolution of longitudinal phase space when the bunch passes through the linac compressor for the optimized result case 1 in Table II. These longitudinal phase spaces are plotted for $90 \%$ of particles with $10 \%$ longitudinal tail cutoff (as explained in the footnote of Table I). These longitudinal phase spaces correspond to the locations at $110 \mathrm{~cm}$ (i.e., the focal point after the rf gun) for plot (a), $334 \mathrm{~cm}$ for plot (b), $414 \mathrm{~cm}$ for plot (c), $504 \mathrm{~cm}$ for plot (d), $675 \mathrm{~cm}$ (i.e., the final point of the linac compressor) for plot (e), and $414 \mathrm{~cm}$ for plot (f) of the lattice in Fig. 4, respectively. In plot (a) to plot (e), the particles are colored according to the energy spread at the focal point before the chicane (i.e., at $110 \mathrm{~cm}$ of the linac compressor shown in Fig. 4). In plot (f), the particles are colored according to the transverse horizontal position at the focal point before the chicane (i.e., at $110 \mathrm{~cm}$ of the linac compressor shown in Fig. 4).

The bunch energy chirp rotates from negative to positive [as shown in Figs. 7(a)-7(f)]; then, after the chicane, in the final focusing section, the chirp reverses its direction of rotation and reaches a very short bunch length [as shown in Fig. 7(e)]. Before the middle of the chicane, the head particle has higher energy as shown in Fig. 7(b). In the

TABLE I. Performances of the BNL rf gun linac compressor and the LBNL's VHF gun linac compressor.

\begin{tabular}{|c|c|c|c|c|c|}
\hline Linac bunch compressor & & $\begin{array}{l}\text { With BNL gun }{ }^{\mathrm{a}} \\
\quad(6.75 \mathrm{~m} \text { long })\end{array}$ & & $\begin{array}{l}\text { With LBNL's VHF gun }{ }^{\text {b }} \\
(8.75 \mathrm{~m} \text { long })\end{array}$ & \\
\hline Bunch performance & & Initial Bunch & $\begin{array}{l}\text { Bunch at the } \\
\text { end of linac } \\
\text { compressor }^{\mathrm{c}}\end{array}$ & Initial Bunch & $\begin{array}{l}\text { Bunch at the } \\
\text { end of linac } \\
\text { compressor }^{\mathrm{c}}\end{array}$ \\
\hline Longitudinal bunch length [fs] & & $1270^{\mathrm{d}}$ & $166^{\mathrm{e}}$ & $6783^{\mathrm{f}}$ & $128^{\mathrm{e}}$ \\
\hline Horizontal beam size $[\mu \mathrm{m}]$ & & $2000^{\mathrm{d}}$ & $31^{\mathrm{e}}$ & $1994^{\mathrm{f}}$ & $42^{\mathrm{e}}$ \\
\hline Vertical beam size $[\mu \mathrm{m}]$ & & $2000^{\mathrm{d}}$ & $28^{\mathrm{e}}$ & $1971^{\mathrm{f}}$ & $25^{\mathrm{e}}$ \\
\hline Energy spread $[\%]$ & $\Delta E / E$ & $0.09^{\mathrm{g}}$ & 0.93 & $0.0014^{g} / 0.98^{h}$ & 1.38 \\
\hline Average kinetic energy [MeV] & E & $4.69^{\mathrm{g}}$ & 4.69 & $0.73^{g} / 22^{h}$ & 22 \\
\hline Horizontal emittance $[\mu \mathrm{m}]$ & $\varepsilon_{x}$ & $0.177^{\mathrm{g}}$ & 1.02 & $59^{g} / 0.143^{h}$ & 0.71 \\
\hline Vertical emittance $[\mu \mathrm{m}]$ & $\varepsilon_{y}$ & $0.189^{\mathrm{g}}$ & 0.84 & $58.5^{g} / 0.142^{h}$ & 0.19 \\
\hline Charge $[\mathrm{pC}]$ & $Q$ & 50 & 50 & 200 & 200 \\
\hline Current $[\mathrm{A}]$ & $I$ & 40 & 300 & 30 & 1563 \\
\hline
\end{tabular}

\footnotetext{
${ }^{\mathrm{a}}$ The schematic is shown in Fig. 1(a).

${ }^{\mathrm{b}}$ The schematic is shown in Fig. 1(c).

${ }^{\mathrm{c}}$ The bunch parameters are calculated for $90 \%$ of particles, with $10 \%$ longitudinal tail cutoff. The particles which are cut off are those particles which have the largest longitudinal position deviation from the average longitudinal position of the bunch at the final point of the linac bunch compressor.

${ }^{\mathrm{d}}$ At cathode: longitudinal distribution is Gaussian with $2 \sigma_{z}=1.27 \mathrm{ps}$; transverse distribution is uniform with the same radius of 2 mm. ${ }^{\mathrm{e}}$ rms value.

${ }^{\mathrm{f}}$ At cathode: longitudinal distribution is flattop with a linear ramp at two ends, and the total length from head to tail is 6.78 ps; transverse distribution is a uniform ellipse with a hard cut edge, and the diameter of the ellipse in $x$ and $y$ is $1.99 \mathrm{~mm}$.

${ }^{\mathrm{g}}$ Downstream of $\mathrm{rf}$ acceleration.

${ }^{\mathrm{h}}$ Downstream of the gun.
} 
TABLE II. Performances of linac compressors with different charge and different energy.

\begin{tabular}{|c|c|c|c|c|c|c|c|c|c|c|}
\hline Case $^{a}$ & Schematics $^{\mathrm{b}}$ & rf gun & Accelerate cavity & $\begin{array}{c}\text { Cavity } \\
\text { length } \\
{[\mathrm{m}]}\end{array}$ & $\begin{array}{c}\text { Compressor } \\
\text { length } \\
{[\mathrm{m}]}\end{array}$ & $\begin{array}{c}\text { Charge } \\
{[\mathrm{pC}]}\end{array}$ & $\begin{array}{l}\text { Energy } \\
{[\mathrm{MeV}]}\end{array}$ & $\begin{array}{l}\sigma_{L}{ }^{\mathrm{c}} \\
{[\mathrm{fs}]}\end{array}$ & $\begin{array}{l}\sigma_{H}^{\mathrm{c}} \\
{[\mu \mathrm{m}]}\end{array}$ & $\begin{array}{c}\sigma_{V}{ }^{\mathrm{c}} \\
{[\mu \mathrm{m}]}\end{array}$ \\
\hline 1 & I & BNL & No & 0 & 6.75 & 50 & 5 & 166 & 31 & 28 \\
\hline 2 & II & rf gun & $\begin{array}{c}2.856 \mathrm{GHz} \text { SLAC-like } \\
\text { cavity }\end{array}$ & 0.95 & 6.75 & 100 & 12 & 110 & 34 & 31 \\
\hline 3 & II & & $\begin{array}{c}2.856 \mathrm{GHz} \text { SLAC-like } \\
\text { cavity }\end{array}$ & 0.95 & 6.75 & 150 & 12 & 145 & 35 & 24 \\
\hline 4 & III & LBNL & $\begin{array}{l}1.3 \mathrm{GHz} \text { TESLA-like } \\
\text { superconducting cavity }\end{array}$ & $2 \times 1.32$ & 8.5 & 150 & 18 & 130 & 47 & 28 \\
\hline 5 & III & $\begin{array}{l}\text { VHF } \\
\text { gun }\end{array}$ & $\begin{array}{l}1.3 \mathrm{GHz} \text { TESLA-like } \\
\text { superconducting cavity }\end{array}$ & $2 \times 1.32$ & 8.5 & 200 & 20 & 148 & 46 & 25 \\
\hline 6 & III & & $\begin{array}{l}1.3 \mathrm{GHz} \text { TESLA-like } \\
\text { superconducting cavity }\end{array}$ & $2 \times 1.32$ & 8.5 & 200 & 22 & 128 & 42 & 25 \\
\hline
\end{tabular}

${ }^{\mathrm{a}}$ The simulation results for the linac compressor with the BNL photocathode rf gun come from PARMELA with CSR effects ignored. The simulation results for the linac compressor with the LBNL's VHF photocathode rf gun come from IMPACT-T with CSR effects considered.

${ }^{\mathrm{b}}$ The schematics are shown in Fig. 1.

${ }^{\mathrm{c}}$ The bunch parameters are calculated for $90 \%$ of particles, with $10 \%$ longitudinal tail cutoff (as explained in the footnote of Table I). $\sigma_{L}, \sigma_{H}$, and $\sigma_{V}$ are the rms value for the $90 \%$ selected particles.

middle of the chicane, the bunch length reaches the maximum as shown in Fig. 7(c). To overcome the drift space's negative $R_{56}$, we need to overcompress the bunch at the third dipole in the chicane as shown in Fig. 7(d). At this position, the initial head becomes the tail, and the energy spread starts to decrease. At the end of the linac bunch compressor, we obtain a compressed and focused bunch as shown in Fig. 7(e). Figure 7(f) shows the longitudinal phase
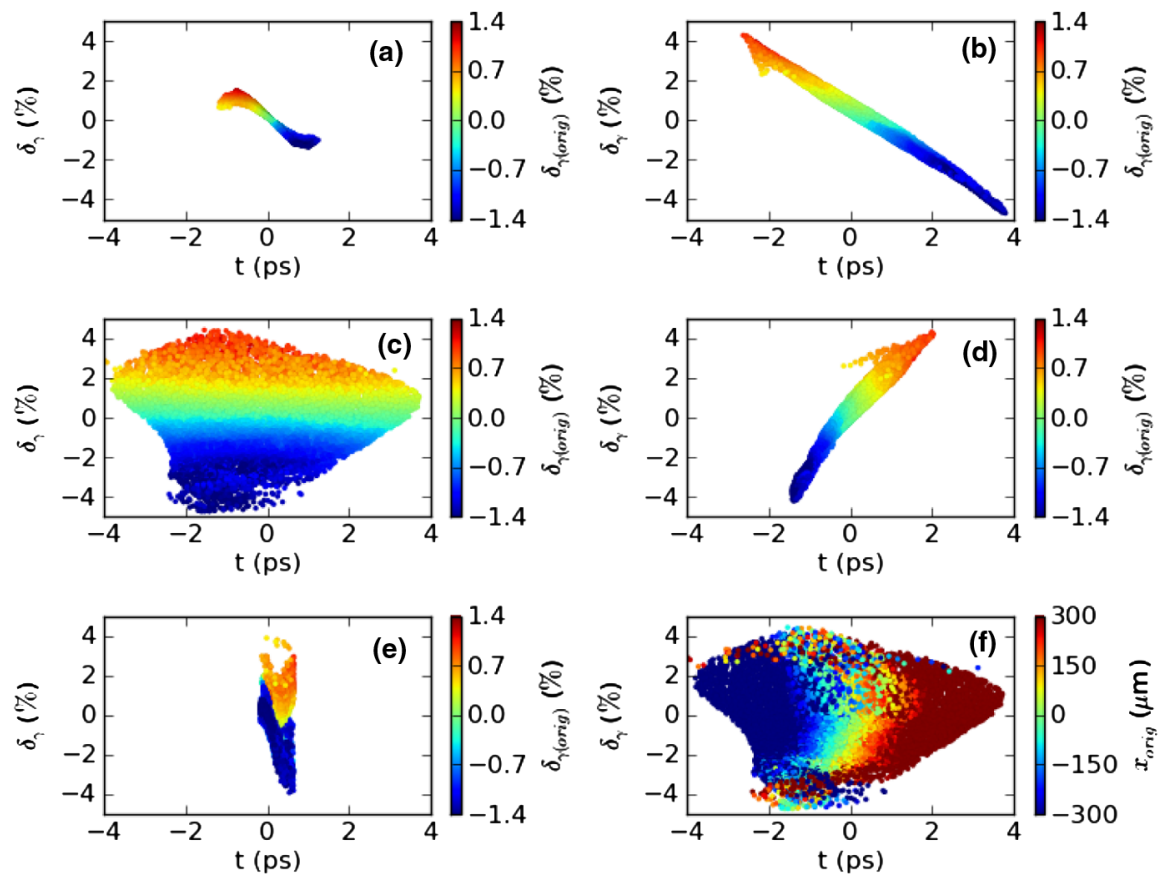

FIG. 7. Evolution of longitudinal phase space during the linac bunch compressor of case 1 in Table II. These longitudinal phase spaces are plotted for $90 \%$ of particles with $10 \%$ longitudinal tail cutoff (as explained in the footnote of Table I). These longitudinal phase space plots correspond to the locations at $110 \mathrm{~cm}$ for (a), $334 \mathrm{~cm}$ for (b), $414 \mathrm{~cm}$ for (c), $504 \mathrm{~cm}$ for (d), $675 \mathrm{~cm}$ for (e), and $414 \mathrm{~cm}$ for (f) of the linac bunch compressor as shown in Fig. 4 respectively. In plot (a) to plot (e), the particles are colored according to the energy spread at the focal point before the chicane (i.e., at $110 \mathrm{~cm}$ of the linac compressor shown in Fig. 4). In plot (f), the particles are colored according to the transverse horizontal position at the focal point before the chicane (i.e., at $110 \mathrm{~cm}$ of the linac compressor shown in Fig. 4). In these plots, particles located at $t>0$ correspond to the tail particles of the bunch. 


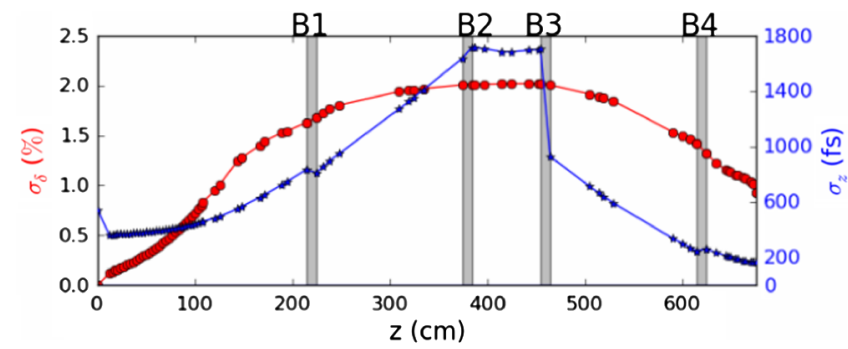

FIG. 8. Evolution of rms energy spread (the red circles) and rms longitudinal bunch length (the blue stars) along the linac bunch compressor of case 1 in Table II. The four gray bars show the four dipole magnets of the chicane compressor. The rms energy spread is calculated by $100 \%$ particles. The rms longitudinal bunch length is calculated for $90 \%$ of particles, with $10 \%$ longitudinal tail cutoff (as explained in the footnote of Table I).

space at the same position as in Fig. 7(c), but the particles are colored according to the particles' horizontal position at $110 \mathrm{~cm}$ of the linac bunch compressor. Figure 7(f) indicates the longitudinal path length spread is due to different initial horizontal position. The optimized result shows the structure of our chicane, and the bunch behaviors in the chicane are partially symmetric relative to the center of the chicane.

The energy spread evolution caused by space charge effects is useful and important in our compressor. When the energy spread increases, the required quadrupole magnet's strength and dipole magnet's strength to bend the trajectory for high energy particles are reduced. Therefore, the particles' trajectory restores to the same trajectory as if there is no space charge as shown in the top plot of Fig. 5. On the other hand, at the final focal point, due to Liouville's theorem, the longitudinal phase space is conserved, and then increased energy spread leads to smaller bunch length. To increase energy spread, we strongly focus the bunch upstream of the chicane compressor in Fig. 7. It is remarkable that the energy spread increase in our compressor is not an irreversible process, as shown in Fig. 8.

\section{BENCHMARK, CSR EFFECTS, AND THE HIGH REPETITION RATE COMPRESSOR}

\section{A. Benchmark and CSR effects}

We applied another tracking code IMPACT-T [12] to benchmark the simulation results of the low energy compressor obtained from code PARMELA. The IMPACT-T code is a three-dimensional (3D) parallel particle-in-cell code for self-consistent beam dynamics simulation. Both linac tracking codes PARMELA and IMPACT-T can track relativistic particles taking into account space charge effects in the 3D space. The 3D space charge model used in both codes is the same; i.e., both solve the 3D Poisson equation subject to free boundary conditions using a FFT method. The difference is that IMPACT-T uses an integrated Green's function method [12] (while PARMELA uses a standard Green's function), which enables it to have better numerical accuracy in solving the Poisson equation in the beam frame when the longitudinal-to-transverse ratio becomes large. The IMPACT-T code also considers the CSR effects which are not included in PARMELA. The 1D CSR model implemented in the IMPACT-T code does not assume an ultrarelativistic approximation but uses an analytical model developed by Murphy, Krinsky, and Gluckstern [19] and Saldin et al. [20], which is the same as what Sagan et al.'s paper has explored [21]. This analytical CSR model was implemented in the IMPACT-T code by using an integrated Green's function method as discussed in Refs. [22,23].

To verify our simulation results obtained from PARMELA, taking the compressor of case 3 in Table II as an example, we benchmark the results with PARMELA against those with IMPACT-T. By applying IMPACT-T, we also discuss the CSR effect which is not considered in code PARMELA. Short bunches on curved trajectories will emit coherent synchrotron radiation. The resulting energy loss varies along the bunch length; it increases energy spread and transverse emittance and may even disturb the compression process itself. The CSR effects are related to the bunch energy, bunch charge, and bunch length. To check the CSR effects, we compare the simulation results when CSR effects are turned on with the results when CSR effects are turned off in IMPACT-T. The results of the benchmark and comparison are shown in Table III and Figs. 9 and 10.

Data in Table III show that the rms bunch length difference between PARMELA and IMPACT-T with CSR off is less than $6 \%$. The difference of the transverse rms beam size between the two codes is larger than $20 \%$ when all the results are calculated for $90 \%$ particles, with $10 \%$ longitudinal tail cutoff at the final point of the linac bunch compressor (as explained in the footnote of Table I). If the transverse rms beam sizes are calculated for $100 \%$ particles as shown in Fig. 9, the difference of those between the two codes decreases to $\sim 10 \%$. Figure 9 shows the longitudinal, horizontal, and vertical phase spaces at the final focus point for the three simulation results: PARMELA (the first row), IMPACT-T with CSR effects turned off (the second row), and

TABLE III. The benchmark results of PARMELA against IMPACT-T and the comparison of CSR turning off with CSR turning on in IMPACT-T. We take the optimized $12 \mathrm{MeV}, 150 \mathrm{pC}$ bunch compressor of case 3 in Table II as an example to do the benchmark and comparison.

\begin{tabular}{lcccc}
\hline \hline Code & CSR effects & $\sigma_{L}{ }^{\mathrm{a}}[\mathrm{fs}]$ & $\sigma_{H}{ }^{\mathrm{a}}[\mu \mathrm{m}]$ & $\sigma_{V}{ }^{\mathrm{a}}[\mu \mathrm{m}]$ \\
\hline PARMELA & Off & 145 & 35 & 24 \\
IMPACT-T & Off & 137 & 45 & 32 \\
IMPACT-T & On & 157 & 41 & 26 \\
\hline \hline
\end{tabular}

${ }^{\mathrm{a}}$ The bunch parameters are calculated for $90 \%$ of particles, with $10 \%$ longitudinal tail cutoff (as explained in the footnote of Table I). $\sigma_{L}, \sigma_{H}$, and $\sigma_{V}$ are the longitudinal rms bunch length, the horizontal rms beam size, and the vertical $\mathrm{rms}$ beam size for the $90 \%$ selected particles, respectively. 
PARMELA

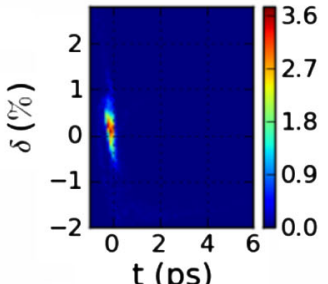
$\mathrm{t}(\mathrm{ps})$

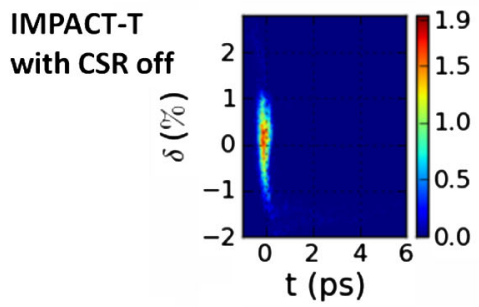

IMPACT-T with CSR on

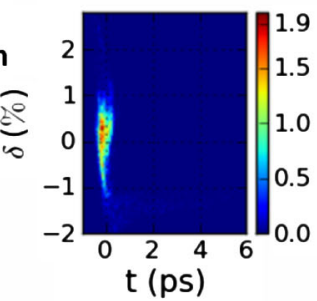

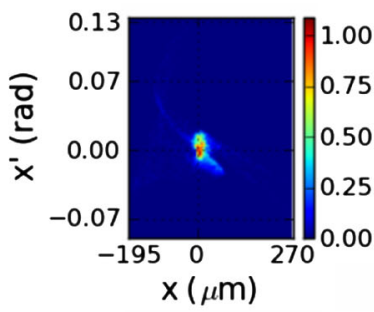
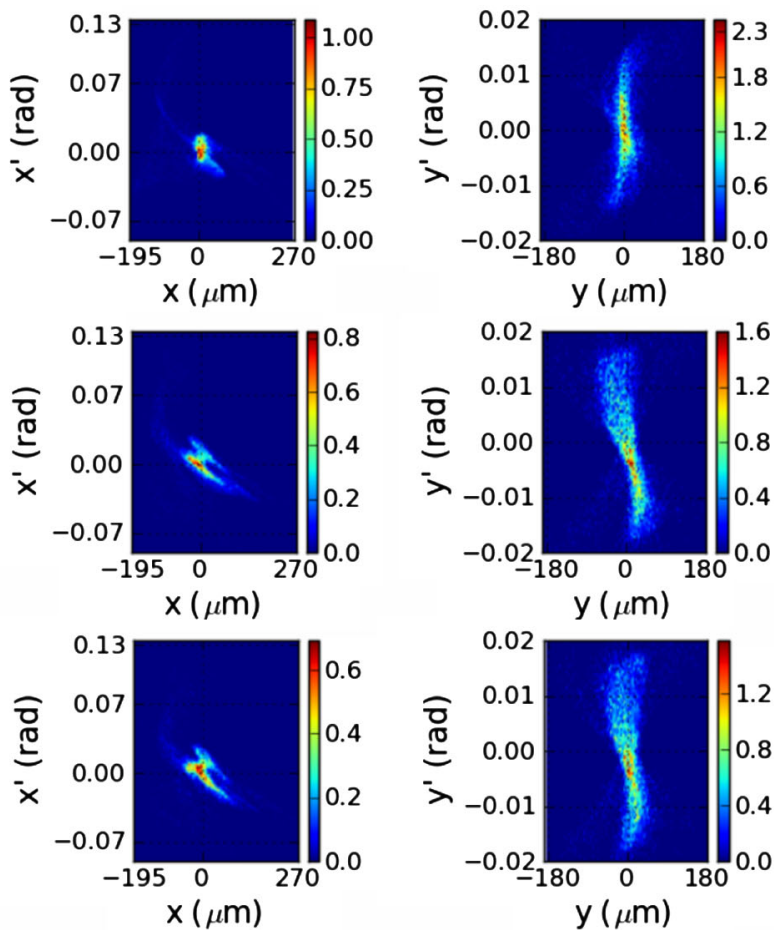

FIG. 9. The histograms of the bunch's longitudinal phase space (the left column), horizontal phase space (the middle column), and vertical phase space (the right column) at the end of the compressor. These plots are plotted for 100\% particles at the final point of the linac bunch compressor. Data come from different simulation codes for the same linac compressor of case 3 in Table II: PARMELA (the first row), IMPACT-T with CSR effects turned off (the second row), and IMPACT-T with CSR effects turned on (the third row). The color bar shows the particle density.

IMPACT-T with CSR effects turned on (the third row). The color bar indicates the particle density.

To show the agreement between the two codes and the difference of the calculations with and without the CSR effects considered, we also plot the evolution of the redefined beta functions and the dispersion function along the dispersive chicane section of the compressor for the three simulation results in Fig. 10. To calculate the redefined beta functions, we select those particles whose emittance before the bending magnet $q B 4$ (at $2.14 \mathrm{~m}$ in Fig. 4) is smaller than $0.1 \mathrm{~mm}$ mrad. Then we use these selected particles' rms beam size as beta functions (actually, we plot the beam size in Fig. 10). To calculate the redefined dispersion, we select those particles whose energy deviation before the bending magnet $q B 4$ (at $2.14 \mathrm{~m}$ in Fig. 4) is in the range $0.7 \%-0.8 \%$. Then we use these selected particles' average trajectory along the compressor as the dispersion function. Although the exact values of these functions in Fig. 10 lose their original meaning with space charge effects increasing, these function curves still can be used to approximately compare the simulation results.

The simulated results of IMPACT-T with CSR off (green curve) show $~ 90 \%$ agreement with those of PARMELA (red curve) for the rms beam size. The agreement between the two codes (the red and green curve) for the dispersion is good. The green curves (CSR off) and the blue curves (CSR on) are completely overlapped before the chicane's third bending magnet, which is located at $4.6 \mathrm{~m}$ of the beam line. The green and blue curves appear separated after the third dipole. The separation indicates the CSR effects and also shows that the CSR effects mainly take place at the third and fourth dipoles of the chicane. Analysis shows that the bunch length is significantly compressed from 800 to $300 \mathrm{fs}$ when passing through the third dipole and the bunch length has been compressed to 235 fs upstream of the fourth dipole. Although the CSR effects induce some minor difference, the results in Table III indicate it is promising that the final bunch can be further optimized.

The benchmark between PARMELA and IMPACT-T has produced a good agreement. The comparison of the CSR effects also shows that the bunch can be compressed and focused to our desired size after optimization using code IMPACT-T with CSR effects turned on.

\section{B. High repetition rate low energy compressor}

In order to increase the repetition rate of the electron beam slicing system to increase the photon flux, we change the rf gun of the linac bunch compressor from the BNL photocathode rf gun [7] to LBNL's VHF gun, which operates at $186 \mathrm{MHz}$ with a repetition rate of $1 \mathrm{MHz}$ [13], and redesign the linac bunch compressor by applying simulation code IMPACT-T with both space charge effects 

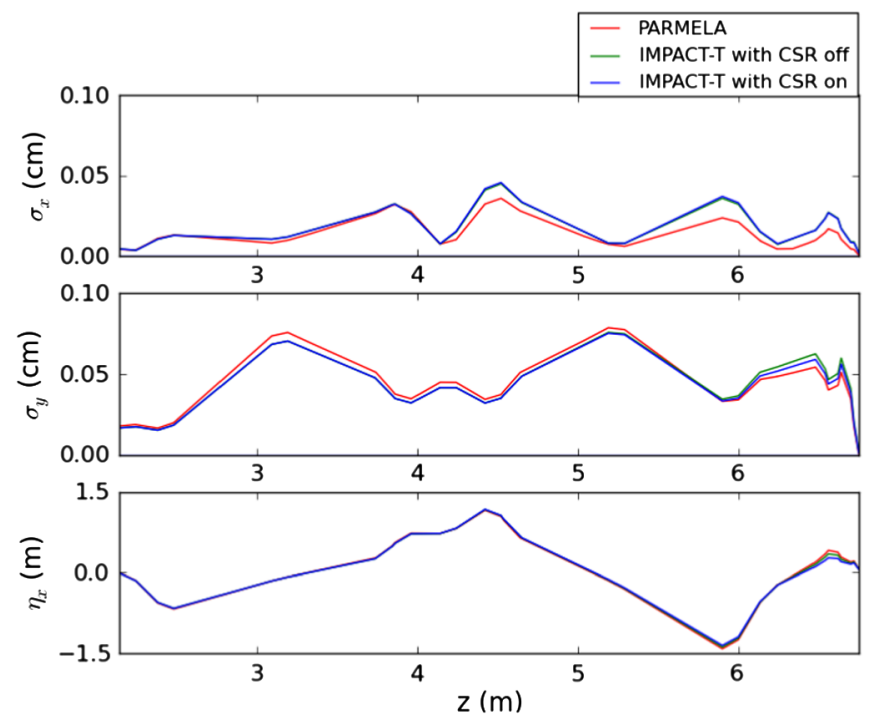

FIG. 10. The evolution of the redefined beta functions and the redefined dispersion function along the dispersive section of the second linac bunch compressor [i.e., Fig. 1(b)]. The red, green, and blue curves correspond to the simulation results by PARMELA, IMPACT-T with CSR effects turned off, and IMPACT-T with CSR effects turned on, respectively.

and CSR effects considered. The schematic for the third linac bunch compressor with LBNL's VHF gun is shown in Fig. 1(c).

\section{Matching by manual adjustment}

Since the design of the low energy linac bunch compressor is a rather tedious and time-consuming optimization process, we would like to design the high repetition rate system starting from the previous successful result rather than starting over from scratch again. Hence, we utilize the simulation result of the $12 \mathrm{MeV}, 150 \mathrm{pC}$ linac bunch compressor (i.e., case 3 in Table II) as a basis to study the linac compressor with LBNL's VHF gun. We change the gun from the BNL photocathode if gun to LBNL's VHF gun, and we also add two $1.3 \mathrm{GHz}$ TESLA-like superconducting cavities between the gun and the compressor section as shown in Fig. 1(c).

At the photocathode of the third linac bunch compressor [i.e., Fig. 1(c)], we set the bunch longitudinal distribution as flattop with a linear ramp at two ends, and the total length from head to tail is $8 \mathrm{ps}$. The bunch transverse distribution is a uniform ellipse with a hard cut edge, and the diameter of the ellipse in $x$ and $y$ is $1.5 \mathrm{~mm}$. The 3D momentum distribution of the bunch is Gaussian. The bunch charge is set at $150 \mathrm{pC}$. The simulation shows that the beam energy after LBNL's VHF gun is $730 \mathrm{keV}$. In order to avoid a large betatron oscillation and the beam blowing up, we move the accelerating cavity very close to the photocathode gun.

While keeping the bunch energy at $12 \mathrm{MeV}$ after acceleration, we adjust the phases and gradients of the rf cavities manually to match the bunch's energy chirp after acceleration with the bunch's energy chirp upstream of the quadrupole magnet $q D 6$ of the $12 \mathrm{MeV}, 150 \mathrm{pC}$ linac bunch compressor (i.e., case 3 in Table II). At the same time, we adjust the intensity of focusing solenoid manually to make the bunch's transverse beam size match with those upstream of the quadrupole magnet $q D 6$ of the $12 \mathrm{MeV}$, $150 \mathrm{pC}$ linac bunch compressor.

We iterate the adjustments of the focusing coils and the rf cavities to optimize the matching. After the matching, the total length of the linac bunch compressor system consisting of the LBNL's VHF gun, two focusing coils, two rf cavities, and the matching, dispersive, and focusing magnets section is $8.5 \mathrm{~m}$. Upstream of the first matching quadrupole magnet (i.e., $q D 6$ ), the bunch's energy spread is increased to $\pm 9 \%$, and the longitudinal bunch length is about 25 ps.

\section{Global optimization by genetic algorithm}

With the matched linac bunch compressor in the previous subsection, we apply the genetic algorithm to optimize the linac bunch compressor with the bunch energy fixed at
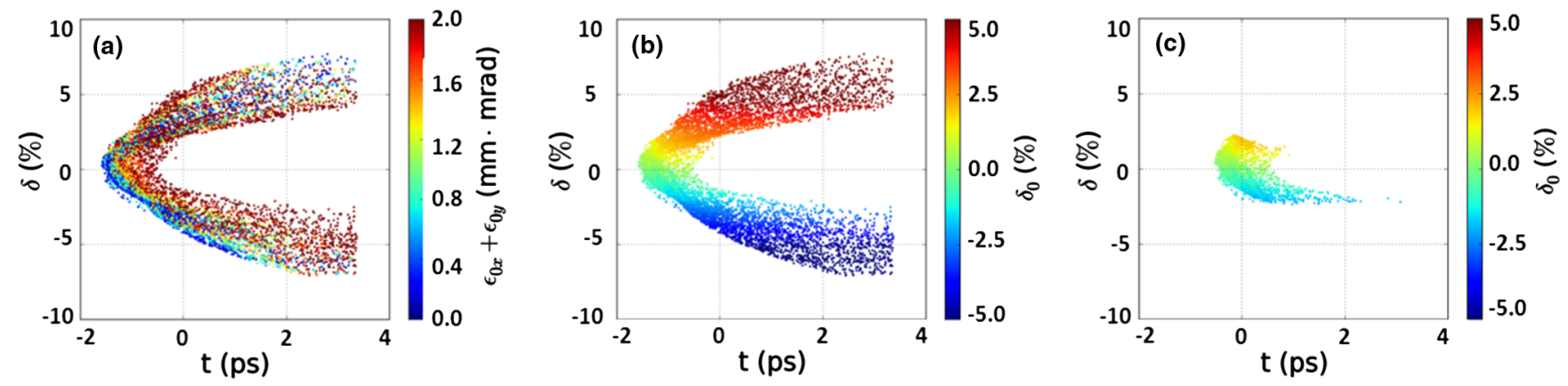

FIG. 11. The longitudinal phase spaces at the final focal point for one optimized result of the linac bunch compressor with LBNL's VHF gun at $12 \mathrm{MeV}$. In plot (a), the particles are colored according to $\varepsilon=\varepsilon_{0 x}+\varepsilon_{0 y}$ downstream of the accelerating cavities; in plot (b), the particles are colored according to the energy spread downstream of the accelerating cavities; in plot (c), the particles with the initial energy spread in the range $-2 \%$ to $+2 \%$ are colored according to the energy spread downstream of the accelerating cavities. The simulation code is IMPACT-T with both space charge effects and CSR effects being turned on. 

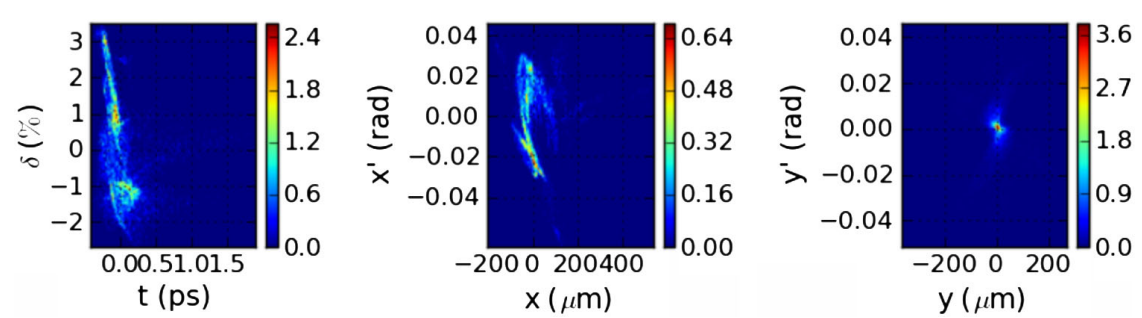

FIG. 12. The histograms of a bunch's longitudinal phase space (the left column), horizontal phase space (the middle column), and vertical phase space (the right column) at the final point of the linac compressor for case 6 in Table II. These plots are plotted for $100 \%$ particles at the final point of the linac bunch compressor. The color bar shows the particle density. The simulation code is IMPACT-T with both space charge effects and CSR effects being turned on.

$12 \mathrm{MeV}$. After optimization, the rms bunch length is still larger than $1 \mathrm{ps}$, and the transverse rms beam size is also very large around $150 \mu \mathrm{m}$. We analyze the bunch's longitudinal phase space at the final point of the linac compressor as shown in Fig. 11. In Fig. 11(a), the particles are colored according to $\varepsilon$ downstream of the rf cavity, where $\varepsilon=2 J$ and $J$ is the action variable. In Fig. 11(b), the particles are colored according to the energy spread downstream of the rf cavity. We find that the bunch length increase is not directly related to the initial $\varepsilon$ downstream of the rf cavity but is related to the initial energy spread (i.e., the energy spread downstream of the rf cavity). If we artificially cut off those bunch particles with the initial energy spread larger than $\pm 2 \%$, the rms bunch length at the final focal point decreases from 1.26 to 0.38 ps as shown in Fig. 11(c).

Based on the previous observation, in order to reduce the final bunch length, we increase the bunch energy to decrease the relative energy spread downstream of the rf cavity acceleration. At the same time, in order to increase the kick angle and shorten the slice width in the electron beam slicing method [6], we also increase the bunch charge. Using IMPACT-T with CSR effects turned on, we iterate to carry out the multiobjective optimization procedure using the genetic algorithm [11] and gradually increase the bunch's charge and the bunch's energy.

Some optimized results are shown in Tables I and II. For an example, the linac bunch compressor with LBNL's VHF gun as shown in Fig. 1(c) longitudinally compresses the $22 \mathrm{MeV}, 200 \mathrm{pC}$ charged bunch from $6.8 \mathrm{ps}$ (this bunch length at the photocathode is measured from the bunch's head to the bunch's tail) to $128 \mathrm{fs}$ rms bunch length. The transverse beam sizes are focused from the diameter ellipse of $2 \mathrm{~mm}$ at the photocathode to 42 and $25 \mu \mathrm{m}$ for horizontal and vertical rms beam size, respectively. Figure 12 shows the histograms of the longitudinal, horizontal, and vertical phase space at the final point of the $22 \mathrm{MeV}$ linac compressor (i.e., case 6 in Table II) with the color bar indicating the particle density.

\section{CONCLUSION AND DISCUSSION}

Our simulation shows that an electron bunch with high charge can be compressed to a very high current bunch at very low energy with space charge effects and CSR effects included.

Bunch lengths of $\sim 100$ fs have been obtained before the final focusing quadrupoles with $200 \mathrm{pC}$ charge. This opens the possibility of using a low energy linac bunch compressor to obtain a very short bunch with low emittance at low energy. We expect that, with some dedicated design and study, it is possible to obtain a smaller energy spread and smaller emittance by using a low energy linac bunch compressor. While this is not useful for the electron beam slicing project, it may be useful for FEL work.

\section{ACKNOWLEDGMENTS}

We appreciate Dr. Simone Di Mitri for discussing the CSR effects and providing the cost estimate of our system's magnets. We acknowledge the support of our colleagues of the accelerator group in NSLS-II BNL. This work was funded by Department of Energy under Contracts No. LDRD12-023 and No. LDRD14-022.

[1] P. Emma, in Proceedings of the 20th International Linac Conference, LINAC-2000, Monterey, CA, 2000 (SLAC, Menlo Park, CA, 2000).

[2] R. Akre, Phys. Rev. ST Accel. Beams 11, 030703 (2008).

[3] H. L. Owen and B. D. Muratori, in Proceedings of the 9th European Particle Accelerator Conference, Lucerne, 2004 (EPS-AG, Lucerne, 2004).

[4] Y. Kim, in Proceedings of the 9th European Particle Accelerator Conference, Lucerne, 2004 (EPS-AG, Lucerne, 2004).

[5] B. E. Carlsten and S. J. Russell, Phys. Rev. E 53, R2072(R) (1996).

[6] A. He, F. Willeke, and L. H. Yu, Phys. Rev. ST Accel. Beams 17, 040701 (2014).

[7] D. T. Palmer et al., in Proceedings of the Particle Accelerator Conference, Dallas, TX, 1995 (IEEE, New York, 1995).

[8] V. Lebedev, OptiM computer code for linear and nonlinear optics calculation, http://www-bdnew.fnal.gov/ pbar/organizationalchart/lebedev/OptiM/optim.htm.

[9] M. Borland, ANL Advanced Photon Source Report No. LS-287, 2000. 
[10] J. H. Billen, PARMILA, Report No. LA-UR-98-4478, 2001.

[11] L. Yang, D. Robin, F. Sannibale, C. Steier, and W. Wan, Nucl. Instrum. Methods Phys. Res., Sect. A 609, 50 (2009).

[12] J. Qiang, Phys. Rev. ST Accel. Beams 9, 044204 (2006).

[13] F. Sannibale et al., Phys. Rev. ST Accel. Beams 15, 103501 (2012).

[14] B. Aune et al., Phys. Rev. ST Accel. Beams 3, 092001 (2000).

[15] M. Dohlus, T. Limberg, and P. Emma, in ICFA Beam Dynamics Newsletter 11-36, 2005.

[16] P. Piot, D. R. Douglas, and G. A. Krafft, Phys. Rev. ST Accel. Beams 6, 030702 (2003).
[17] R. J. England, J. B. Rosenzweig, G. Andonian, P. Musumeci, G. Travish, and R. Yoder, Phys. Rev. ST Accel. Beams 8, 012801 (2005).

[18] S. Y. Lee, Accelerator Physics, 3rd ed. (World Scientific, Singapore, 2012), Eq. (2.98).

[19] J. B. Murphy, S. Krinsky, and R. L. Gluckstern, Part. Accel. 57, 9 (1997).

[20] E. L. Saldin, E. A. Schneidmiller, and M. V. Yurkov, Nucl. Instrum. Methods Phys. Res., Sect. A 398, 373 (1997).

[21] D. Sagan, G. Hoffstaetter, C. Mayes, and U. Sae-Ueng, Phys. Rev. ST Accel. Beams 12, 040703 (2009).

[22] J. Qiang, C. E. Mitchell, and R. D. Ryne, Nucl. Instrum. Methods Phys. Res., Sect. A 682, 49 (2012).

[23] C. E. Mitchell, J. Qiang, and R. D. Ryne, Nucl. Instrum. Methods Phys. Res., Sect. A 715, 119 (2013). 